\title{
Understand, respect and restore anatomy as close as possible!
}

\author{
John Feagin ${ }^{2}$ Michael T. Hirschmann ${ }^{1} \cdot$ Werner Müller $^{1}$
}

Received: 21 January 2015 / Accepted: 4 May 2015 / Published online: 17 May 2015

(C) European Society of Sports Traumatology, Knee Surgery, Arthroscopy (ESSKA) 2015

In the early years of ACL surgery, disappointing clinical outcomes after ACL surgery were observed. One study by Feagin and Curl investigated the clinical 5-year outcomes after ACL repair surgery in cadets [3]. This study was the initial spark and driving force for increasing our efforts to achieve better results after ACL surgery. The ACL study group was founded in 1978, which continually focuses on the scientific exchange and debate regarding ACL surgery, rehabilitation and injury prevention.

Since the early years of knee surgery, time has passed, but the basic principles still remain the same. As knee surgeons, we should strive to "Understand, respect and restore anatomy as close as possible!".

Anatomy is the basis and surgery in its best is applied anatomy. Outcomes after knee surgery are only as good as our knowledge of anatomy and our understanding of knee function. This is particularly true for patients suffering from complex knee instability. When performing reconstructive knee surgery, a deep anatomical and functional understanding allows us to translate our knowledge into surgical reality. It is not the surgical technique per se, which is the most decisive factor for successful outcomes and good function, but the question if such a technique closely restores the patient's individual anatomy, while not harming other structures.

Michael T. Hirschmann

Michael.Hirschmann@ksbl.ch

1 Department of Orthopaedic Surgery and Traumatology, Kantonsspital Baselland (Bruderholz, Liestal, Laufen), 4101 Bruderholz, Switzerland

2 Duke Sports Medicine Center, Box 3371, Finch Yeager Building 280 Frank Bassett Drive, Durham, NC 27710, USA
During the last century, the understanding of knee function has undergone a tremendous change and development. Numerous abstracts, publications and presentations have dealt with the optimal diagnostic assessment and treatment of patients with complex knee instability. Since our active time, a considerable number of new ideas and innovative surgical techniques have been proposed; some were successful and others less so. However, a mistake can easily happen and it is our duty for the sake of our patients not to repeat the same mistake again. Learning comes from experience, and thus, we have to remember previous failures when rethinking reconstructive knee surgery.

We have come a long way. We began with open knee surgery. It was the time of extensive open-book approaches. We were driven by our constant doubts about what we did. At this time, numerous techniques were proposed for ligamentous reconstruction. Many of them failed and did not restore normal knee function-these did not respect anatomy.

Recognising that the ACL and PCL form a four-bar linkage system was a major finding in the past. This rolling and gliding of the femur was essential for the understanding of the pivot-shift mechanism [6]. A tear of the ACL disrupts the four-bar linkage system and breaks the rolling-gliding mechanism down [5]. The femoral condyle rolls too far backwards and then snaps forward again into the demanded correct physiologic position [5].

The era of open anatomical ligament reconstructions began. This lasted until the introduction of arthroscopy in our daily clinical practice. On the way from open to arthroscopic surgery, some obvious anatomical facts, known by open knee surgeons, were neglected for the good of technical feasibility and comfort [1]. The transtibial techniques were scarcely able to put the tunnels in anatomical positions. Finally nowadays, after the detour of double-bundle 
reconstructions, most people perform single-bundle anatomical ACL reconstructions, as we did 30 years ago [1].

What else is new? The anterolateral ligament has been rediscovered [2]. This broadened the knee surgeon's perspective from the ACL as a sole structure towards a more holistic approach. It has brought back to our attention the complex interplay of ACL, PCL, collaterals, menisci and peripheral structures.

However, not every innovation really is one. In a time in which PubMed listing differentiates the valuable from other publications, it is worthwhile not to forget older textbooks $[2,4,6]$. There is a high chance that someone else has already described the newly discovered anatomical structure or novel surgical technique.

Many surgeons think because a surgical technique is new, it must be better than older ones for the same condition-but this is not always the case.

The latest technique and method may not always be the best one to use. When a surgical technique has only been available for a short time, there can be reasons to be more cautious about using it. Our knowledge of benefits and functional outcomes grows only with time.

In the following theme issue, we have the pleasure to present a large number of fantastic papers dealing with knee function and complex knee instability. We hope you enjoy reading this theme issue and keep your eyes wide open for future advancements in reconstructive knee surgery.

Finally, we would like to finish with a quote from a famous British novelist and 2007 Nobel Prize winner in literature, Doris Lessing: "That is what learning is. You suddenly understand something you have understood all your life, but in a new way".

\section{References}

1. Arnold MP, Friederich NF, Muller W, Hirschmann MT (2013) From open to arthroscopic anatomical ACL-reconstructions: the long way round. A statement paper. Knee Surg Sports Traumatol Arthrosc 21(7):1478-1481

2. Claes S, Vereecke E, Maes M, Victor J, Verdonk P, Bellemans J (2013) Anatomy of the anterolateral ligament of the knee. J Anat 223(4):321-328

3. Feagin JA Jr, Curl WW (1976) Isolated tear of the anterior cruciate ligament: 5-year follow-up study. Am J Sports Med 4(3):95-100

4. Feagin JA (1988) The crucial ligaments: diagnosis and treatment of ligamentous injuries about the knee. Churchill Livingstone, London

5. Mueller W (1977) Lesions of the cruciate ligaments. Zentralbl Chir 102(16):974-981

6. Mueller W (1982) The knee: form, function, and ligament reconstruction. Springer, Berlin 